\title{
ه Eau et Santé - L'eau polluée le plus grand fléau du monde
}

\author{
- Water and Health - Polluted water, the greatest calamity in the world - \\ Docteur Loïc Monjour \\ Association EAST
}

(Eau, Agriculture et Santé en Milieu Tropical)

\section{Les infections liées à l'eau de boisson sont très nombreuses dans les pays tropicaux. Mais l'installation de forages ne peut améliorer la situation, que si elle est accompagnée de mesures d'hygiène.}

\section{E INTRODUCTION}

Cette moitié de siècle aura vu la Science révolutionner nos modes de vie et notre conception du monde. Mais ce génie inventif, qui tend à abolir le temps et l'espace, et qui s'acharne à décrypter notre patrimoine génétique, aura fait bien peu de cas des pollutions et dégradations de l'environnement. Toutefois, depuis peu, sous la pression des politiques et des médias, prévention des risques toxiques et infectieux et protection des ressources deviennent des termes d'actualité. Le public montre un engouement pour l'écologie. Il déclare vouloir faire des efforts pour préserver l'hygiène de son milieu ambiant et respecter la nature.
Sans être grand devin, il est sûr que dans les prochaines années, des efforts devront être faits, surtout dans les pays tropicaux, en matière de qualité de l'eau.

Pour bien vivre, en bonne santé physique, les sociétés humaines doivent bénéficier d'une eau de boisson sans contaminants pathogènes. C'est le cas en France, où diton, les inondations font plus de victimes que les maladies infectieuses d'origine hydrique. En fait, la notion de salubrité n'est pas nouvelle. Déjà, dans l'Antiquité, les romains s'inquiétaient de la nocivité de l'eau du Tibre et, par de gigantesques aqueducs, délivraient de l'eau de source, plus saine, à raison de 200 litres par habitant et par jour. L'idée d'une transmission des maladies, par l'intermédiaire de l'eau de boisson, était donc déjà acquise. Mais, curieusement, une vraie conception de l'hygiène hydrique ne sera développée qu'au début du siècle, précédée, cependant, par 3 événements essentiels. La découverte, en 1785, par Claude Berthollet -un chimiste français- de l'hypochlorite de sodium, qui donnera naissance à l'eau de Javel, le désinfectant universel. L'invention par John Gibb, en 1804, à Paisley, en Ecosse [1], du filtre à sable lent, réputé pour délivrer une eau claire et débarrassée de ses contaminants pathogènes. Enfin, l'ac-

WHO estimates that about 20 million people die in the world, each year, due to drinking water-related diseases. The absence of safe water and sanitation, specially in developping countries plays a major part in this tragedy: Bacteriological analysis of 1700 water points in West Africa revealed that $100 \%$ of ponds, $70 \%$ of rural wells, $15 \%$ of concrete-lined wells and $7 \%$ of boreholes, contain water unsafe for human consumption. So, installation of boreholes, found to be the best safeguard against the bacterial contamination of water, has to be developed in African rural areas. However, from boreholes to consumer, safe drinking water usually becomes polluted with foecal bacteria during transport and storage. For bacteriological decontamination, sterilisation in the UV or by the use of traditional filters has been proposed and affords no guarantee for the health of the consumer. In contrast, chlorination represents a final option for the purification of drinking water associated with improved environmental sanitation. Finally; carefully planned health education is of fundamental importance within the context of rural hydraulic programmes having the power to mobilise the population for the promotion of safe drinking water improving health and greater well-being. 
tion décisive de Louis Pasteur, le plus grand hygiéniste de son époque, qui sera le promoteur de la chloration de l'eau de consommation humaine.

Aujourd'hui, dans les pays nantis, les infections liées à l'eau de boisson sont exceptionnelles. En revanche, dans les pays tropicaux, en particulier dans les zones périurbaines et rurales, la situation est préoccupante et le pire est à venir en raison de la forte expansion démographique.

\section{II " $\mathrm{C}$ 'HOMME BOIT $80 \%$ DE SES MALADIES" DANS LES PAYS DU TIERS-MONDE}

Cette déclaration de Pasteur, déplorant la médiocrité des conditions d'hygiène en Europe, au siècle dernier, s'applique toujours aux pays pauvres. L'Organisation Mondiale de la Santé (OMS) ne cesse de renforcer les inquiétudes. Elle estime qu'en un an 20 millions de personnes disparaissent de notre planète en raison de la pollution pathogène de l'eau et de l'insalubrité de l'environnement. Le signe pathologique majeur de ce manque d'hygiène est la diarrhée -ou gastroentériteinfectieuse. Il existe, par jour, 600 millions de cas de gastroentérites dans le monde et le volume des diarrhées est comparable au débit minute d'eau des Chutes Victoria sur le Zambèze, soit 40 à $60 \mathrm{~m}^{3} / \mathrm{seconde}$. La fantaisie de cette estimation s'estompe, quand apparaissent les conséquences. Au bilan des décès, s'ajoute une longue litanie de maladies microbiennes (choléra, shigelloses, salmonelloses...), virales (hépatites, poliomyélite...) et parasitaires (amibiase, giardiase, dracunculose...), incapacitantes et invalidantes $[2,3]$. Elles limitent la productivité de la main d'œuvre, contribuent à l'absentéisme scolaire et représentent une lourde charge économique pour la communauté. En termes de gestion, elles font de pauvres pays, des pays encore plus pauvres.

Ici peut s'exprimer, encore, le décalage entre les Etats du Nord et du Sud. Si nous bénéficions d'une eau potable, incolore, inodore et sans saveur, la ration quotidienne des villageois, dans les pays tropicaux, n'est qu'un cocktail turbide pathogène renfermant, bien souvent, des milliers de bactéries mortelles.

\section{LA QUALITÉ DE L'EAU DE BOISSON EN MILIEU TROPICAL}

Face à cette situation très préoccupante, les Nations Unies lançaient, en 1977, lors de la Conférence de Mar del Plata, la "Décennie Internationale de l'Eau Potable et de l'Assainissement (DIEPA : 1981-1990)". Les objectifs étaient les suivants : fournir à tous au moins 20 litres d'eau potable par jour ; faire bénéficier les populations d'installations de salubrité. Ces objectifs ne seront pas tenus. Dans l'échelle d'importance des pathologies majeures et terrifiantes -comme les cancers, le paludisme et le SIDA - les gastroentérites infectieuses occupent. toujours, la première place, comme en attestent les centaines d'articles scientifiques publiés depuis 1990, après la Décennie $[4,5,6,7,8,9,10 \ldots]$.

Il est vrai, qu'a priori, l'eau des forages protégée par les structures géologiques du sol, pouvait apparaître sans danger pour l'homme. Ce raisonnement, pourtant, ne tient pas si l'on se réfère aux résultats d'une enquête, menée par l'Association EAST (Eau, Agriculture et Santé en milieu Tropical), au Burkina Faso [11]. Mille sept-cent points d'eau bénéficiaient d'une analyse bactériologique effectuée par la méthode de la membrane filtrante [12, 13]. Et le pourcentage des ressources polluées, contenant de l'eau impropre à la consommation humaine (selon les Normes internationales de l'OMS : > à 0 coliformes fécaux et 10 coliformes totaux/100 ml) était le suivant : mares $(100 \%)$, puits traditionnels $(70 \%)$, puits aménagés $(15 \%)$, forages $(7 \%)$.

Cette étude débouchait sur 2 conclusions : le puits traditionnel est un véritable danger public : le forage, muni de sa pompe hydraulique, représente le meilleur équipement de prévention contre la souillure hydrique. Et, pourtant, l'eau de $7 \%$ des forages s'avérait insalubre en raison de 3 causes essentielles : les imperfections des pompes et le mauvais entretien du matériel ; les altérations des sites d'exhaure de l'eau, piétinés et fissurés par le bétail, qui s'abreuve à la pompe ; enfin, le manque d'hygiène alentour des points d'eau.

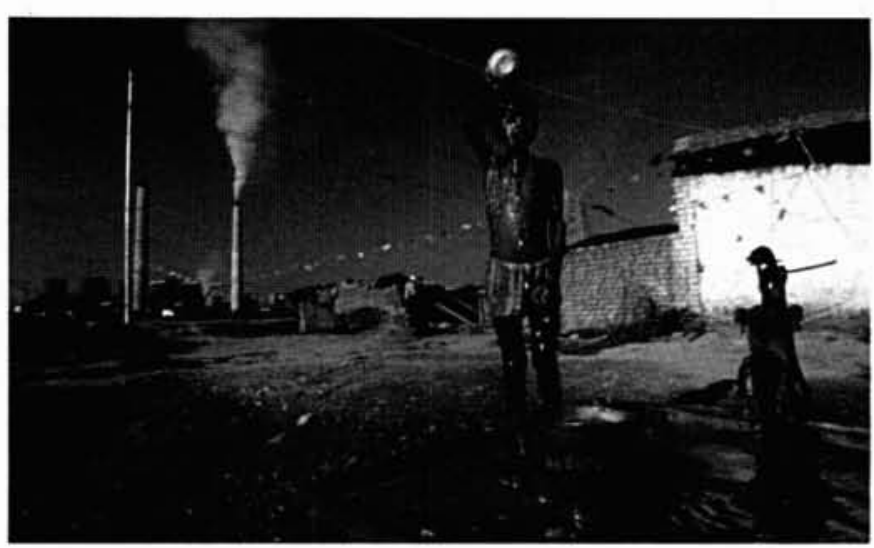

Ces observations devaient déboucher sur une liste de recommandations, mais une seconde enquête, menée, récemment, par EAST, décelait un autre problème, plus inquiétant [3]. Elle avait pour objectif l'évaluation de la qualité de l'eau de boisson domestique en milieu rural. Potable au bec de la pompe, elle apparaissait, progressivement, contaminée par des bactéries d'origine fécale lors de son transport pour devenir un véritable bouillon de culture microbien dans les jarres de stockage. Ainsi, les villageois, qui bénéficient d'un forage, consomment une eau aussi dangereuse pour leur santé - avec plus de 4000 coliformes fécaux/litre - que celle du puits traditionnel. 
La situation est critique et paradoxale ; la quasi totalité du monde rural tropical ou subtropical boit de l'eau insalubre, vit en état de diarrhée chronique, mais a peu d'accès aux soins ; en revanche, le bétail, qui s'abreuve à l'orifice de la pompe, consomme de l'eau potable, dont sont dépourvues les communautés villageoises.

Pourquoi, dès lors, préconiser l'installation d'ouvrages hydrauliques modernes, dont le coût unitaire est particulièrement élevé, de l'ordre de 40000 - 50000 F ? Pour 2 raisons essentielles : la première est l'insuffisance des ressources disponibles. Dans les régions soudano-sahéliennes d'Afrique de l'ouest, par exemple, les puits tarissent à la saison sèche et le nombre de forages est de 1 pour 2000 habitants. La seconde est la qualité de l'eau émise au bec de la pompe. Claire, limpide, sans matières en suspension, elle se prête au mieux à la chloration. Il faut donc encourager l'implantation de forages et initier, dans les plus brefs délais, de larges programmes d'éducation sanitaire et de désinfection de l'eau. En fait, la priorité des femmes en milieu tropical, - ignorantes des relations causes-effets des pollutions hydriques, surchargées de tâches domestiques et d'activités agricoles - est l'élimination de la fatigue causée par le transport de l'eau. Elles se servent, donc, au plus près des habitations, quelle que soit la salubrité de la ressource : mare, puits ou forage. A présent, deux objectifs sont envisagés : l'implantation d' 1 forage pour 300 habitants et le renforcement de l'éducation sanitaire dans les villages. Ces mesures, qui constituent des priorités, devraient déboucher sur une amélioration des conditions de vie et de santé en milieu rural $[4,7,8]$.

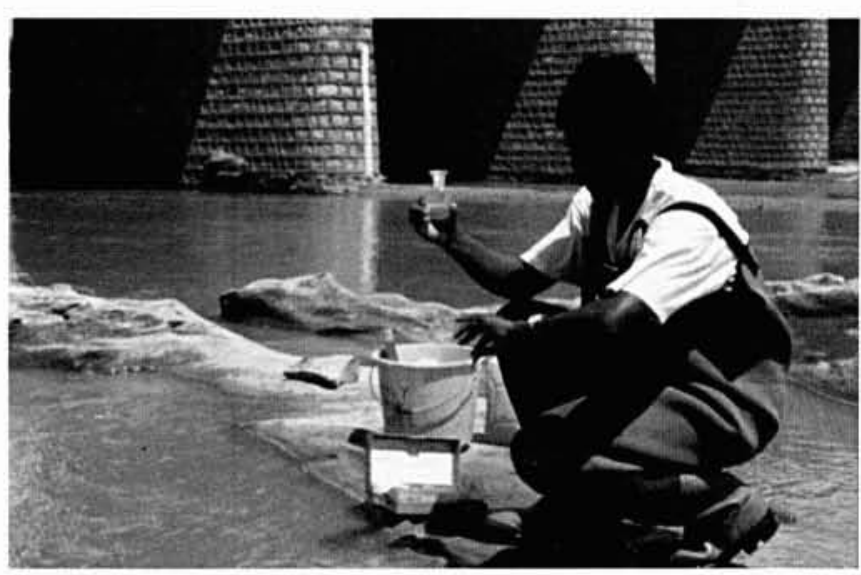

\section{IV a PRÉVENTION ET TRAITEMENT DE LA POLLUTION DE L'EAU DE BOISSON}

L'euphorie manifestée à l'échéance de la "Décennie Internationale de l'Eau Potable et de l'Assainissement" (DIEPA) s'est, peu à peu, transformée en un optimisme de circonstance [14], sous la pression des médecins de Santé publique, qui considèrent la DIEPA comme un semi-échec. La composante sanitaire, disent-ils, a été ignorée ou négligée, l'objectif s'étant concentré sur le nombre de puits ou forages à réaliser en un temps donné.
De plus, étaient omises, bien souvent, des programmes, la contrainte du contrôle de la qualité bactériologique de l'eau et la promotion de l'éducation sanitaire. Le personnel médical, depuis lors, ne cesse de repéter que le bénéfice pour la santé humaine de l'eau potable demeure un symbole majeur. C'est l'un des seuls qui puisse entraîner l'adhésion des populations, le développement de l'hygiène et la maintenance des ouvrages d'hydraulique villageoise. La consommation d'une eau potable ne peut résulter, cependant, que de décisions opportunes :

\section{Construire, réhabiliter et protéger les points d'eau}

Si l'on se réfère à l'Afrique de l'ouest, le nombre de points d'eau modernes, en particulier de forages, n'est pas suffisant pour satisfaire les besoins quantitatifs des villageois. Atteindre le ratio 1 forage/300 habitants, réhabiliter ceux qui sont en panne, assurer la protection des aires d'exhaure et rénover les puits modernes, représentent les priorités d'aujourd'hui dans les programmes d'hydraulique rurale. On peut améliorer leur fréquentation à deux conditions : augmenter le nombre d'ouvrages ; les présenter comme un symbole d'acquisition d'une meilleure santé. A la première condition, s'attache l'organisation de structures de maintenance : création de Comités de gestion des points d'eau bien informés de leur rôle ; formation et équipement de mécaniciens locaux chargés de la réparation des puits et des pompes ; enfin, mise en place d'un réseau de pièces détachées étendu et achalandé. La seconde condition implique une coopération étroite entre responsables de l'hydraulique et animateurs spécialistes de l'éducation sanitaire. L'appui des animateurs peut favoriser les
Le contrôle de la qualité de négociations portant sur le financement de l'entretien des ouvrages par les bénéficiaires. l'eau en Inde sur le site de Singrault (Inde).

(Photothèque EDF Photo Georges Mérillon).

\section{Eduquer la population-cible}

C'est un élément-clef, irremplaçable $[4,7,8]$, le starter de la réussite des campagnes d'hydraulique rurale. Dans un contrat d'installation de puits ou de forages, le volet "Education sanitaire" doit s'imposer, ne plus être un paragraphe vide à l'extrême fin d'un document. Car peu de succès est à attendre sans une prise de conscience de l'intérêt de l'eau potable pour la santé.

Les éducateurs sanitaires sont, tout d'abord, des formateurs. Usant de matériels et de méthodes pédagogiques (exemple : méthode GRAAP), appropriés à la compréhension d'une population peu instruite ou analphabète, ils s'appliquent à répondre aux souhaits d'informations concrètes sur les maladies, leurs causes et leurs conséquences. Ils rappellent et démontrent aux villageois la nécessité des soins, de la prévention et l'importance de la consommation d'une eau saine. Eduquer, persuader, motiver, requiert un haut degré de compétence et des spécialistes en relations humaines. De plus, comme rien n'est acquis, à court terme, par une sen- 
sibilisation à l'emporte -piéce, l'immersion des éducateurs en milieu rural est indispensable. Elle peut perdurer pendant plusieurs mois.

Responsabiliser des villageois qui, de tous temps, ont opposé une certaine inertie au développement socio-sanitaire implique, aussi, la création d'Offices départementaux de conseils, de contrôle et de prévoyance. Ces structures décentralisées, en contact direct avec l'habitant, connaissant ses habitudes et ses traditions - de plus aptes à résoudre ses problèmes administratifs ou logistiques peuvent apporter des bénéfices incontestables aux programmes d'hydraulique rurale. Multiplier les sessions d'éducation sanitaire est la première mesure à préconiser, mais doivent, impérativement, s'y ajouter la désinfection de l'eau et l'assainissement.

\section{Promouvoir la désinfection de l'eau et l'assainisse- ment}

Fourniture par un équipement adéquat d'eau saine ne se traduit pas par consommation permanente d'eau potable. Si plus de $90 \%$ des forages délivrent une eau de bonne qualité, elle est systématiquement contaminée par des bactéries d'origine fécale, pendant son transport et son stockage. Aussi, afin d'éliminer les fortes pollutions pathogènes hydriques, la désinfection de l'eau est une étape obligatoire. Plusieurs études, menées en milieu rural africain, ont souligné les désavantages des procédés de traitement mécanique et physique [15]. La filtration rapide sur sable, à l'aide de filtres dits traditionnels, n'est pas fiable ; parfois, elle concentre, au mieux, les bactéries dans l'eau de boisson [16]. La désinfection, par les rayons UV solaires, est anecdotique, dépendant de la qualité des récipients transparents et des conditions météorologiques [17, 18, 19]. Enfin, l'ébullition ne peut être retenue, en particulier dans les régions soudano-sahéliennes, en raison de la déforestation. Il faut compter $1 \mathrm{~kg}$ de bois pour amener un litre d'eau à ébullition. Reste la désinfection chimique, et principalement, la chloration, dont l'usage est généralisé dans le monde. Ses performances la rendent irremplaçable dans les zones rurales des pays tropicaux.

Le produit générateur de chlore, le plus adéquat, est l'eau de Javel [20]. Peu toxique après dilution [21, 22, 23], son activité microbicide est remarquable. Elle agit tout au long du chemin de l'eau, au cours du transport et du stockage, pendant 18 heures. De plus, elle inactive les germes, introduits dans l'eau, après la désinfection initiale. Ce résultat, en aucun cas, ne peut être obtenu par des procédés physico-mécaniques de traitement.

En milieu villageois tropical, la chloration par l'eau de Javel, méthode simple, peut s'enseigner lors des sessions d'éducation sanitaire. Et après les démonstrations pratiques, le traitement peut s'appliquer aux réserves d'eau familiales ou communautaires (écoles, dispensaires, associations villageoises...). Il faut, pour protéger le consommateur contre les souillures hydriques bactériennes - selon les recommandations de l'OMS et des traités d'hygiène sanitaire - des concentrations en Chlore Résiduel Libre
(CRL) de l'ordre de $0,5 \mathrm{mg} / \mathrm{l}[24,25]$. Elles peuvent être augmentées - à l'exemple de New York, ville phare de la côte Est américaine - jusqu'à $2 \mathrm{mg} / \mathrm{l}$ [20], pour renforcer la sécurité dans les villages particulièrement insalubres. Peu onéreuse (dépense familiale $=15 \mathrm{~F} / \mathrm{an}$ ), la chloration devrait être largement vulgarisée. Mais la diffusion commerciale de l'eau de Javel s'arrête, bien souvent, aux limites des villes, négligeant le milieu rural.

Les programmes d'approvisionnement en eau potable ne peuvent être dissociés des actions d'assainissement [4. $5,6,9,10]$. Sinon, éparpillés à tous vents, les excrétats humains et animaux polluent les ressources hydriques, parfois jusqu'à la nappe phréatique. Aussi, les constructions de latrines, d'enclos de parcage du bétail et d'aires de destruction des déchets sont à associer aux futurs programmes d'hydraulique villageoise.

En définitive, sans modification des comportements ce qui réclame un vrai savoir-faire en matière d'éducation pour la santé et l'hygiène- sans apprentissage de la chloration et sans un contrôle de l'hygiène de l'environnement, on ne peut s'attendre à la diminution des grandes endémo-épidémies mondiales liées à l'eau de boisson.

Une rapide prise de conscience est impérative, autant des politiques, des décideurs que des usagers. La qualité de l'eau doit devenir la première priorité. Sinon le pire est à envisager. A la moitié du siècle prochain, quand la population mondiale avoisinera - dit-on -12 milliards d'habitants, les gastroentérites infectieuses, aussi galopantes que la démographie, feront disparaître, en 6 ans, près de 250000000 de personnes, une population équivalente à celle des USA. Gageons que ce scénario n'est qu'une utopie, mais ne le laissons pas, cependant, se réaliser.

\section{RÉFÉRENCES}

[1]. Baker M.N. The question for pure water. American waterworks Association. New York, 1949.

[2]. Monjour L.. Towrne F. Problèmes de santé en milieu sahélien. PUF. techniques vivantes. $1980,126 \mathrm{p}$.

[3]. Monjour L. Eau potable et santé : actions prioritaires en milieu tropical. Colloque scientifique et technique international. Hydrotop, Marseille, $12-15$ avril 1994.

[4]. Aziz K.M. Reduction in diarrhoeal diseases in children in rural Bangladesh by environmental and behavioural modificiations. Trans. R. Soc. Trop. Med. Hyg. 1990, $84: 433-438$.

[5]. Huttly S.R. The Imo State (Nigeria) Drinking Water Supply and Sanitation Project 2. Impact on dracunculiasis, diarrhoea and nutritional status. Trans. Roy. S. Trop. Med. Hyg. 1990, 84 : 316-321.

[6]. Gorter A.C. Water supply, sanitation and diarrhoeal disease in Nicaragua : results from a case-control study. Int. J. Epidemiol. 1991, 20 : 527553.

[7]. Ahmed N.U. A longitudinal study of the impact of behavioural change intervention on cleanliness, diarrhoeal morbidity and growth of children in rural Bangladesh. Soc. Sci. Med. 1993, $37: 159-171$.

[8]. Haggerty P.A. Community-based hygiene education to reduce diarrhoeal disease in rural Zaire : impact of the intervention on diarrhoeal morbidity. Int. J. Epidemiol. 1994, 23 : 1050-1059. 
[9]. Messou E. Water supply, excrement disposal and diarrhoeal diseases in rural zones of Ivory Coast. Med. Trop. 1995, $55: 225-230$.

[10]. Messou E. Effect of hygiene measures, water sanitation and oral rehydratation therapy on diarrhea in children less than five years in the south of Ivory Coast. Bull. Soc. Pathol. Exot. 1997, 90:44-47.

[11]. Guillemin F., Henry P., Uwechue N., Monjour L. Faecal contamination of rural water supply in the sahelian area. Water Res, 1991, 25:923. 927.

[12]. Monjour L., Henry P., Guillemin F., Spinasse A., Lagneaux F., Alfred C., Colin J.J., Gentilini M. Etude comparative de méthodes de recherche de la pollution hydrique, d'origine fécale, en milieu rural soudano-sahélien. Bull. Soc. Pathol. Exot? 1986, $79: 540-556$.

[13]. Monjour L., Henry P., Guillemin F. Comparative study of field tests for the detection of polluted drinking water in Sahel regions. Trans. Roy. Soc. Trop. Med. Hyg. 1986, $80: 172-173$.

[14]. Organisation Mondiale de la Santé. Progrès de la décennie internationale de l'eau potable et de l'assainissement, 1981-1990 : huit ans d'activité, Document EB 83/3/1988. OMS éd.

[15]. Monjour L. Relations entre chloration de l'eau et santé. L'actualité chimique, Nov, $1994: 46-51$.

[16]. Monjour L., Volta Ch., Uwechue N., de Lorenzi G. Evaluation of traditional filters for water purification in Burkina Faso. Ann. Soc. Belge Med. Trop. 1990, $70: 311-315$
[17]. Rice E.W., Hoff J.C. Inactivation of Giardia lamblia cysts by ultraviolet irradiation. Appl. Environ. Microbiol. 1981, 42: 546-547.

[18]. Acra A., Raffoul Z., Karahagopian M. Solar desinfection of drinking water and oral rehydratation solution. UNICEF, Beyrouth, 1984, 56 p.

[19]. De Lorenzi G., Volta Ch., Monjour L. Application de la désinfection solaire à l'eau de boisson en milieu tropical. Bull. Soc. Pathol. Exot. 1989. $82: 255-259$.

[20]. Mesny M. Sécurité, eau potable et chloration. Colloque scientifique et technique international. Hydrotop, Marseille, 12-15 avril 1994

[21]. Hartemann E., Hermier M., Ducloux E., Morgon A. et coll. Les intoxications par produits toxiques chez l'enfant. Pédiatrie. 19789, 33: 6171

[22]. Levêque B. L'eau de Javel et ses risques pour l'enfant. Le Concours Médical. 1981, 103:6391-6394.

[23]. Garnier R., Efthymiou M.L., Riboulet-Delmas G. et coll. Contaminations par l'eau de Javel chez l'enfant. J. Toxicol. Med. 1982, 2 : 25-31.

[24]. Organisation Mondiale de la Santé. Directives de qualité pour l'eau de boisson., vol. 2. Critéres d'hygiène et documentation à l'appui. OMS, Genéve, 1986, 341 p.

[25]. Lanoix J.N., Roy M.L. Manuel du technicien sanitaire. OMS, Genéve, 1976. 193 p. 